Article

\title{
Time-Constrained Nature-Inspired Optimization Algorithms for an Efficient Energy Management System in Smart Homes and Buildings
}

\author{
Ibrar Ullah ${ }^{1, *}$ and Sajjad Hussain ${ }^{2}$ (] \\ 1 Department of Electrical Engineering, Capital University of Science and Technology, \\ Islamabad 44000, Pakistan \\ 2 School of Engineering, University of Glasgow, Glasgow G12 8QQ, UK; Sajjad.Hussain@glasgow.ac.uk \\ * Correspondence: ibrarullah@uetpeshawar.edu.pk; Tel.: +92-333-905-1548
}

Received: 14 December 2018; Accepted: 15 February 2019; Published: 23 February 2019

\begin{abstract}
This paper proposes two bio-inspired heuristic algorithms, the Moth-Flame Optimization (MFO) algorithm and Genetic Algorithm (GA), for an Energy Management System (EMS) in smart homes and buildings. Their performance in terms of energy cost reduction, minimization of the Peak to Average power Ratio (PAR) and end-user discomfort minimization are analysed and discussed. Then, a hybrid version of GA and MFO, named TG-MFO (Time-constrained Genetic-Moth Flame Optimization), is proposed for achieving the aforementioned objectives. TG-MFO not only hybridizes GA and MFO, but also incorporates time constraints for each appliance to achieve maximum end-user comfort. Different algorithms have been proposed in the literature for energy optimization. However, they have increased end-user frustration in terms of increased waiting time for home appliances to be switched ON. The proposed TG-MFO algorithm is specially designed for nearly-zero end-user discomfort due to scheduling of appliances, keeping in view the timespan of individual appliances. Renewable energy sources and battery storage units are also integrated for achieving maximum end-user benefits. For comparison, five bio-inspired heuristic algorithms, i.e., Genetic Algorithm (GA), Ant Colony Optimization (ACO), Cuckoo Search Algorithm (CSA), Firefly Algorithm (FA) and Moth-Flame Optimization (MFO), are used to achieve the aforementioned objectives in the residential sector in comparison with TG-MFO. The simulations through MATLAB show that our proposed algorithm has reduced the energy cost up to $32.25 \%$ for a single user and $49.96 \%$ for thirty users in a residential sector compared to unscheduled load.
\end{abstract}

Keywords: energy management system; energy optimization techniques; genetic algorithm; moth-flame optimization; smart grid; time-constrained optimization techniques

\section{Introduction}

Energy utilization efficiency is increasing with increased use of technology and smart appliances in every field of life in the residential, commercial and industrial sectors. At the same time, a reliable and high-quality electrical power system is extremely vital to fulfil the residential energy demand. Meanwhile, there is a rapid increase in demand for global natural resources. Throughout the world, major blackouts occur due to consumer demand and utility supply mismatch and system automation deficiencies. Hence, a transition process from the Traditional Electric Power Grid (TEPG) to the Smart Grid (SG), to integrate communication and information technologies, is the demand of the future. Presently, about $40 \%$ of the total generated energy is consumed by residential users, and approximately $30-40 \%$ of carbon emission is due to these residential areas [1]. The unnecessary and inefficient use of electrical energy brings sustainability issues to the forefront, such as economic growth, heavy 
pollution and global warming. Conventionally, the service provider power systems run on fossil fuel and add to global warming with high carbon emissions. Furthermore, in the present power systems, electricity power flow is uni-directional, i.e., from the supply- to the demand-side. Conversely, SG's purpose is to make the flow of electricity supply and demand bidirectional [2]. Secondly, the search for and integration of new green renewable energy resources are obligatory in such circumstances. The integration of green renewable energy resources needs a broader perspective of design, planning and optimization. Up to this time, different conventional optimization techniques, such as Linear Programming (LP) [3], Non-Linear Programming (NLP) [4], Integer Linear Programming (ILP) [5], Mixed Integer Linear Programming (MILP) [6], Dynamic Programming (DP) [7] and Constrained Programming $(\mathrm{CP})$, have been practised. However, in the present situations, the integration of renewable energy resources is mandatory, and the problems are non-linear and have numerous local optima, making conventional optimization techniques obsolete. In the last decade, bio-inspired modern heuristic optimization techniques have grown in popularity due to their stochastic search mechanisms and avoidance of large convergence time for the exact solution [1].

In this research work, we propose a new meta-heuristic optimization algorithm, named Time-constrained Genetic-Moth-Flame Optimization (TG-MFO), and applied it for efficient energy optimization in smart homes and buildings. We have also explored and analysed five bio-inspired heuristic algorithms for the energy optimization problem, namely the ACO, GA, Cuckoo Search Algorithm (CSA), Firefly Algorithm (FA) and MFO algorithms. For analysis and validation of the proposed algorithm, we applied these algorithms in different consumer scenarios, such as a single home for one day, a single home for thirty days, thirty different sizes of homes for one day and thirty homes for thirty days. Simulation results show that our proposed algorithm reduced the end-user discomfort in terms of appliance waiting time being nearly equal to zero, as compared to the bio-inspired optimization algorithms, along with minimization of total energy cost and minimum PAR. Renewable energy sources are also integrated for further minimization of the total load and its cost.

To achieve this goal, the smart electric grid is modelled as a residential sector comprised of 30 homes having different sizes, different Lengths of Operational Time (LOTs) and appliance power ratings. Appliance power ratings are different due to the home size requirements. For example, a small-sized home runs a one-ton (12,000 BTU) air conditioner compared to a large-sized home that runs 1.5 tons (18,000 BTU) or even more. Some homes have a Renewable Energy Source (RES) and a Battery Storage Unit (BSU). In the considered model, we have forty-eight (48) Operational Time Intervals (OTIs) in a day, by dividing one hour into two-time slots of thirty minutes each. In each OTI, a smart home checks appliances' power demand, i.e., whether an appliance is ON or OFF. According to the appliances' ON/OFF status, the Energy Management Controller (EMC) checks the availability of RES and BSU to fulfill the appliances' power demand. If it is available, the appliance will be ON, and the consumer will not wait for appliance scheduling. If the generation and stored energy are insufficient for running the load, the proposed algorithm will check the time span, in which a user has no problem with appliance scheduling with the lowest energy price (time interval) for running that appliance. In order to achieve this objective, time constraints have been defined for a maximum interval of time in which an appliance has to complete its operation. Consequently, if the utility gives incentives to the user in the form of real-time lower prices in off-peak hours, the end-user will be encouraged to produce his/her own energy from RES and schedule the load accordingly. The rest of the paper is organized as follows: Related work is illustrated in Section 2. Contributions are briefly discussed in Section 3. Section 4 depicts the proposed system model architecture. The problem formulation is described in Section 5, and Section 6 gives the heuristic algorithms. Section 7 presents the simulation results to demonstrate some of the achievements. The paper is concluded in the last section. 


\section{Related Work}

Countless researchers around the world are investigating different technologies in order to fulfill the needs of energy-efficient and intelligent smart homes. Many algorithms have been proposed for optimal use of existing energy resources. In this regard, we illustrate some prior research works in SG.

Yi Peizhong et al. [8] have proposed the Optimal Stopping Rule (OSR) for energy-efficient scheduling of home appliances. The limitation of this work is that OSR runs on a threshold-based strategy. The end-user has to wait until the price comes down below the threshold level. In [9], the authors have proposed an approach to optimize their objective function using the Genetic Algorithm (GA). Electricity prices are varying between on-peak hours and off-peak hours. Therefore, an optimized task scheduling module is used in smart homes, which can reduce the consumption of the entire energy and operation times. Having an optimal scheduling of power, a heuristic-based GA was used for Demand-Response (DR) in Home Energy Management (HEM) systems in [10]. The authors proposed GA-, TLBO- (Teaching Learning-Based Optimization), EDE (Enhanced Differential Evolution) and EDTLA- (Enhanced Differential Teaching Learning-based Algorithm) based approaches, which are used for minimization of the residential total energy cost and maximization of the end-user comfort level.

The problem of optimal scheduling of household appliances has been explored in [11]. The authors used the day-ahead changeable peak pricing technique for the minimization of the consumer's energy consumption cost using a combination problem approach. This approach enables customers to schedule their household appliance using MKP (Multiple knapsack problem) formulation. In [12], the authors implemented GPSO (Gradient-based Particle Swarm Optimization) for DR in smart homes by considering load and energy price uncertainties. The authors employed GA and BPSO (Binary Particle Swam Optimization) for optimal scheduling of home appliances in [13]. They proposed GAPSO (Genetic Algorithm with Particle Swam Optimization), a hybrid scheme of both these techniques, to obtain better results in terms of reducing PAR, minimization of electricity cost and especially end-user discomfort. Day-Ahead Pricing (DAP) and Critical Peak Pricing (CPP) are used as pricing schemes for single and several days. The authors used GA and TLBO and their hybrid TLGO (Teacher Learning-based Optimization with Genetic algorithm) for appliance scheduling in [14]. They categorized flexible appliances as time flexible and power flexible for proficient energy consumption of consumers in SG. This approach enables energy consumers to schedule their appliances to obtain optimized energy consumption. This approach also maximizes the comfort level of customers with restricted total energy consumption. The authors in [15] discussed the strategy for scheduling appliances in order to reduce carbon emissions along with the reduction of the electricity bill and waiting time. They applied the cooperative multi-swarm PSO technique to achieve their goals; however, they did not consider PAR. The authors implemented the $0 / 1$ multiple knapsack problem with the genetic algorithm to find a good solution in [16]. A simple fitness function is evaluated for each appliance in every time slot to obtain the desired results. The authors proposed a Demand-Side Management (DSM) strategy. This technique is based on a load shifting strategy during peak hours to reduce electricity bills using an Evolutionary Algorithm (EA). The authors discussed the strategy of the load shifting-based generalized technique, from on-peak hours to off-peak hours of a day, to minimize energy cost. This mechanism can support a large number of controlled devices of numerous types to minimize end-user electricity bills.

An adaptive energy model for DSM in smart homes has been proposed by the authors in [17]. Distributed RESs' usage is optimized using the ACO algorithm. In [18], Kusakana et al. used the TOU pricing model along with the integration of RESs and BSUs to minimize the end-user's electricity bill and achieve energy consumption balancing. The authors proposed a model to sale extra generated energy back to the utility, as per their prior agreement. For minimization of the end-user electricity bill, Bharathi et al. suggested a model in [19], which works in industrial, commercial and residential areas. For optimization, the authors used GA. They also compared the different EA with GA and found that it gave a maximum decrease of $21.9 \%$ in the consumption of energy. 
In [20], the authors proposed objective function generalization using the DR program to minimize the residential consumer electricity bill. The authors showed that by shifting the load, unexpected peaks were observed in off-peak hours. They evaluated this later peak formation with multi-CPP and multi-TOU pricing schemes combined with DAP concepts. In [21], in order to lessen the end-user electricity cost and minimize the end-user discomfort, Ogunjuyigbe et al. developed a GA-based optimization technique for scheduling of appliances.

In [22], authors presented a DSM strategy, by shifting the load from on-peak hours to off-peak hours, using DAP signal and Evolutionary Algorithm (EA). However, consumer comfort is not considered. In [23], the authors proposed a Quality of Experience (QoE)-based home energy management system. They gave the priority to the end-user's frustration. Two algorithms that run the HEM system are: "QoE-aware Cost Saving Appliance Scheduling (Q-CSAS)" for scheduling of controlled load and "QoE-aware Renewable Source Power Allocation (Q-RSPA)" for management of appliances for renewable energy sources' surplus energy. They reduced energy cost to 30-33\% without RES and $43-46 \%$ with RESs for the end-user annoyance rates of 1.67-3.36 and 1.70-3.43, respectively. In [24], the authors introduced three heuristic-based algorithms: GA, ACO and BPSO, to maximize user comfort, minimize PAR and minimize electricity cost, as well. In [25], the authors proposed a hybrid GA-PSO, which is a combination of the GA and PSO algorithm, for energy management and obtaining maximum end-user comfort in smart homes. K. Muralitharan et al. [26] presented multi-objective EA for the minimization of electricity bill and appliances waiting time. As soon as the running appliances' load increases from a threshold, they are switched off. A multi-residential energy scheduling issue with multi-class appliances in a smart grid was discussed in [27]. The authors proposed a PL-generalized Benders algorithm (Property (P) and L-Dual-Adequacy) for bill minimization and bounded user comfort. In [28], the authors proposed a distributed algorithm for shifting the load from on-peak hours to off-peak hours. They used the game theory approach for scheduling the residential load. The Nash equilibrium convergence rate was also accelerated by the Newton technique. PAR and end-user discomfort were minimized.

The aforementioned research works achieved the cost minimization and reduction of PAR at the cost of end-user's waiting time. Therefore, in this paper, using TG-MFO, we achieved a nearly-zero waiting time along with cost and PAR minimization.

Table 1 depicts the achievements and limitations of the aforementioned research work. 
Table 1. Critical analysis of the related work. OSR, Optimal Stopping Rule; TLBO, Teaching Learning-Based Optimization; DAP, Day-Ahead Pricing; GPSO, Gradient-based Particle Swarm Optimization; TLGO, Teacher Learning-based Optimization with Genetic algorithm; CPP, Critical Peak Pricing; CP, Constrained Programming.

\begin{tabular}{|c|c|c|c|}
\hline Mechanisms/Techniques & Objectives/Requirements & Achievements & Limitations \\
\hline Threshold based OSR [8] & Minimization of electricity bills. & Reduced cost. & Threshold-based cost minimization. \\
\hline GA [9] & Minimization of electricity bills. & Reduced electricity bill. & No PAR is considered. \\
\hline GA, TLBO [10] & Minimization of PAR and electricity bills. & Reduced cost and PAR with RES. & No end-user comfort priority. \\
\hline MKPwith DAP [11] & Minimization of total energy and electricity bill. & Reduced power consumption and electricity bill. & Less end-user comfort level. \\
\hline GPSO with DR [12] & Minimization of PAR and electricity bills. & PAR and minimization of electricity bill. & $\begin{array}{l}\text { No end-user comfort level and no RES and } \\
\text { congestion problem. }\end{array}$ \\
\hline MKP with GA, PSO and GAPSO [13] & Minimization of total energy and electricity bill. & Minimization of electricity bill. & Less end-user comfort level and no RES. \\
\hline GA and TLGO algorithm [14] & Cost minimization plus congestion control. & Minimization of electricity bill. & Waiting time increased. \\
\hline PSO [15] & Optimization of the appliances. & Carbon emission, bill and waiting time minimization. & Less end-user comfort level and no RES. \\
\hline 0/1 MKP and GA [16] & Minimization of PAR and electricity bills. & Minimization of PAR and peak load shifting. & Less end-user comfort level and no RES. \\
\hline ACO [17] & Distributed RES usage is optimized. & Minimization of PAR and peak load shifting. & End-user comfort has been compromised. \\
\hline TOU along with RES and BSUs [18] & To minimize the end-user electricity bill. & Energy consumption balancing. & User comfort has been compromised. \\
\hline GA-based DSM [19] & $\begin{array}{l}\text { Bill minimization for industrial, commercial and } \\
\text { residential consumers. }\end{array}$ & Compared with EA, $20.9 \%$ reduction in bill. & End-user comfort has been compromised. \\
\hline DR with CPP and ToU [20] & $\begin{array}{l}\text { Objective function generalization using the DR } \\
\text { program to minimize bill. }\end{array}$ & Minimized residential consumer electricity bill. & End-user comfort is ignored. \\
\hline GA [21] & $\begin{array}{l}\text { Bill minimization keeping consumers' maximum } \\
\text { satisfaction. }\end{array}$ & Managed the load as per end-user budget. & PAR has been compromised. \\
\hline EA with DAP [22] & Energy bill minimization. & Bill is minimized. & User comfort has been compromised. \\
\hline Q-CSAS and Q-RSPA [23] & Energy bill minimization. & Bill is minimized. & User comfort has been compromised. \\
\hline GA, ACO and BPSO [24] & Cost minimization plus congestion control. & Minimization of electricity bill. & Less end-user comfort level. \\
\hline GA-PSO [25] & $\begin{array}{l}\text { Bill minimization keeping consumers' } \\
\text { maximum satisfaction. }\end{array}$ & Minimized electricity bill. & Less end-user comfort. \\
\hline Multiobjective EA [26] & $\begin{array}{l}\text { Minimization of electricity bill and appliances } \\
\text { waiting time. }\end{array}$ & Minimized electricity bill and appliances waiting time. & Appliances' interruptions increased. \\
\hline PL-generalized Benders algorithm [27] & Multi-residence and multi-class appliance. & $\begin{array}{l}\text { Bill minimized with upper and lower bounds on } \\
\text { user comfort. }\end{array}$ & No RESs and BSUs are integrated. \\
\hline Game theory, Nash equilibrium [28] & $\begin{array}{l}\text { Used distributed algorithm, minimization of PAR } \\
\text { and discomfort. }\end{array}$ & PAR and end-user discomfort have been minimized. & No RES integration. \\
\hline
\end{tabular}




\section{Key Contributions}

The key contributions of this research work are summarized as follows:

- We have proposed a new hybrid end-user comfort-based TG-MFO algorithm for an efficient EMS in smart homes.

- We have explored and analysed the performance of five bio-inspired algorithms for the energy optimization problem in the residential sector.

- Through simulations, we have shown that using TG-MFO, the energy cost can be reduced up to 49.96\% compared to existing methods, with nearly-zero end-user discomfort and PAR up to $60 \%$.

- We have integrated RESs and BSUs for further minimization of the total load and its cost.

- We have applied the proposed algorithms on different consumer scenarios, such as:

(a) A single home for one day,

(b) A single home for 30 days,

(c) Thirty different sizes of homes for one day and

(d) Thirty different sizes of homes for 30 days, compared to the existing techniques.

- We have considered different sizes of homes with different power ratings of appliances and different LOTs as compared to earlier techniques, which are applied on either one home or multiple homes with the same lifestyle.

\section{Proposed System Model}

\subsection{Architecture}

In SG, in order to obtain a reliable and efficient operation, DSM is used. Two main objectives of DSM are end-user controlling activities and energy management. A Smart Home (SH) consists of a smart meter along with the EMC, for a reliable bi-directional power and information flow between SG and SH [29]. All appliances, connected sensors, RESs and BSUs are connected to EMC through a Home Area Network (HAN), which is further connected to SG through a Wide Area Network (WAN). Different WAN solutions are available like PLC, Wi-Fi, Wi-Max and GSM [30]. End-users manage their energy consumption activities as per incentives offered by the utilities. In each $\mathrm{SH}$, the end-user puts various parameters of all appliances in EMC. EMC is then responsible for the ON/OFF status of all appliances. Figure 1 shows the proposed system model architecture.

\subsection{Appliances' Categorization and Their Energy Models}

TO design an optimized model, we have divided the load according to the end-user's priorities, as given below.

\subsubsection{The Fixed Load}

These are those regular appliances whose starting time remains fixed. That is, a consumer can start and stop these appliances any time. Refrigerator and interior lights are examples of Fixed Load (FL). The energy consumed by all fixed appliances in the total time interval of $24 \mathrm{~h}$ can be found as in Equation (1) [24].

$$
\begin{gathered}
E_{f}=\sum_{a p_{f} \in A P_{f}}\left(\sum_{n=1}^{48}\left(\rho_{f} \times X_{f, n} \times \tau_{f}\right)\right) \\
\text { subject to: } \quad \alpha_{f} \leq \tau_{f} \leq \beta_{f}
\end{gathered}
$$

where " $a p_{f}$ " denotes each fixed appliance, $A P_{f}$ is the set of fixed appliances, $\rho_{f}$ is the power rating, $X_{f, n}$ is the ON (1) and OFF (0) states of the nth fixed appliance, $\tau_{f}$ is the LOT, $\alpha_{f}$ is the earliest starting time and $\beta_{f}$ is the latest ending time of fixed appliances, respectively. 
Most of the research works have considered 24 intervals for their energy calculations, which are usually not applicable to all appliances. Usually, an appliance, for example a microwave oven, a clothes dryer, etc., operates for less than an hour. Hence, further dividing an hour into two sub-intervals of thirty minutes each generates a total of forty-eight (48) time slots, which results in accurate manipulation. However, this slightly increased the manipulation time, but that can be ignored.

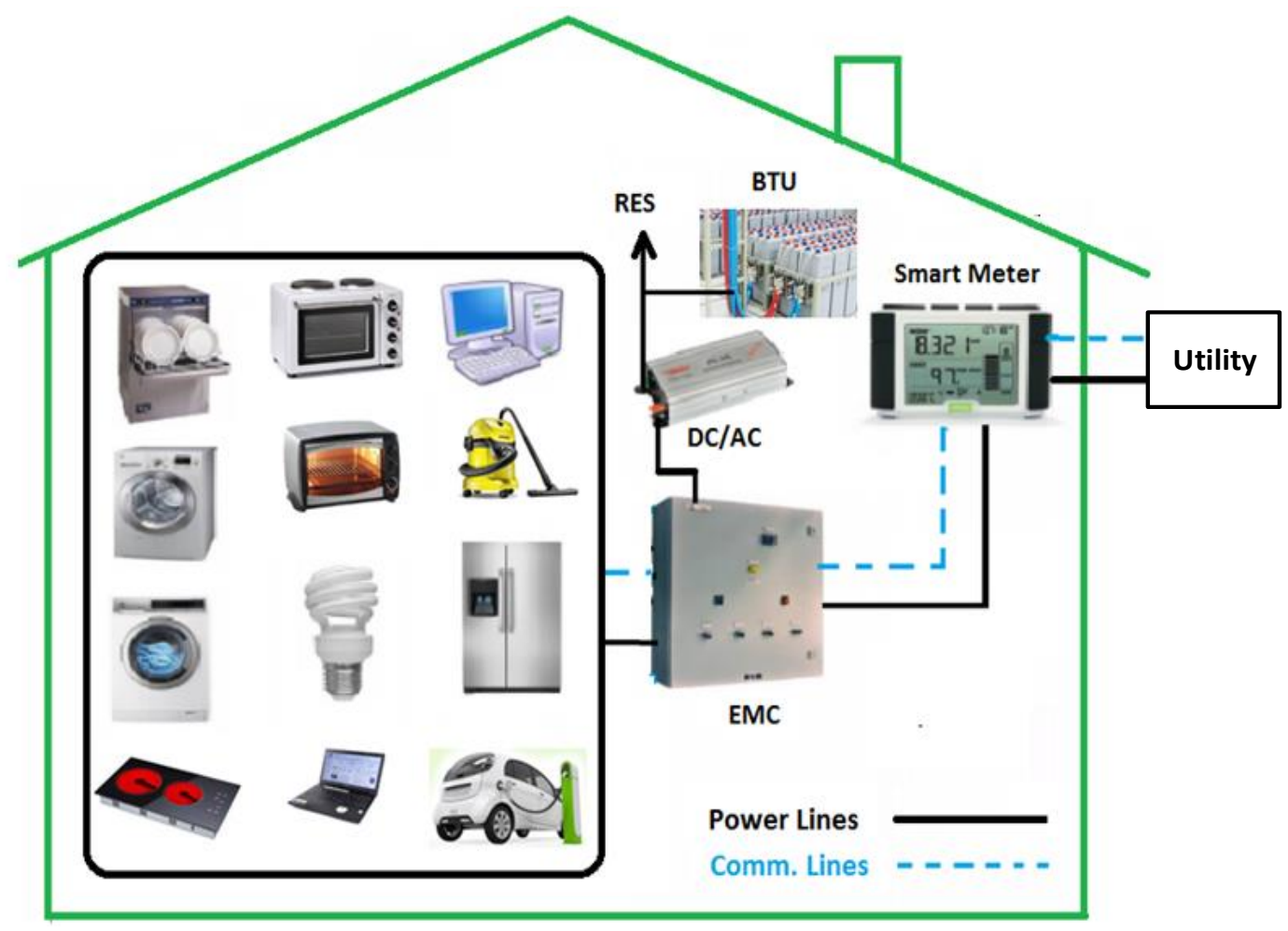

Figure 1. A typical overview of a house with smart appliances. EMC, Energy Management Controller.

\subsubsection{Elastic Load}

These are those appliances that can be fully managed, i.e., they can be shifted to any time slot and can also be interrupted at any time keeping in view the minimization of PAR and electricity bill [24]. These include: dish washer, washing machine, spin dryer, electric car, laptop, desktop computer, vacuum cleaner, oven, cook top and microwave oven [31]. The energy consumed by all elastic appliances in the total time interval of $24 \mathrm{~h}$ with 48 time-slots can be found as in Equation (2).

$$
\begin{gathered}
E_{e}=\sum_{a p_{e} \in A P_{e}}\left(\sum_{n=1}^{48}\left(\rho_{e, n} \times X_{e, n} \times \tau_{e}\right)\right) \\
\text { subject to: } \quad \alpha_{e} \leq \tau_{e} \leq \beta_{e}
\end{gathered}
$$

where $a p_{e}$ denotes each elastic appliance, $A P_{e}$ is the set of elastic appliances, $\rho_{e}$ is the power rating of elastic appliances, $X_{e, n}$ is the ON (1) and OFF (0) states of the $\mathrm{n}^{\text {textth }}$ elastic appliance, $\tau_{e}$ is the LOT for each elastic appliance, $\alpha_{e}$ is the earliest starting time and $\beta_{e}$ is the latest ending time of elastic appliances, respectively. The total energy consumption of the end-user " $E_{T}$ " is given by:

$$
E_{T}=E_{f}+E_{e}
$$

where " $E_{f}$ " is the fixed appliances total energy and " $E_{e}$ " is the total energy of the elastic appliances. 


\subsection{RES Model}

Photovoltaic (PV) cells and wind turbines can be used as local power generators, also known as distributed RESs, on consumer premises. These RESs can be used for the local energy generation, as well as for charging the batteries in BSUs. The RESs' generated energy, denoted by $E_{R E S}$, can be calculated as in [32], by approximating a local Gaussian function (Figure 2) as follows:

$$
E_{R E S}(t, \mu, \sigma)=\frac{1}{\sigma \sqrt{2 \pi}} e^{\left(-\frac{(t-\mu)^{2}}{2 \sigma^{2}}\right)}
$$

where $t$ denotes the prospection variable (time), $\mu$ is the mean or central value and $\sigma$ is the standard deviation.

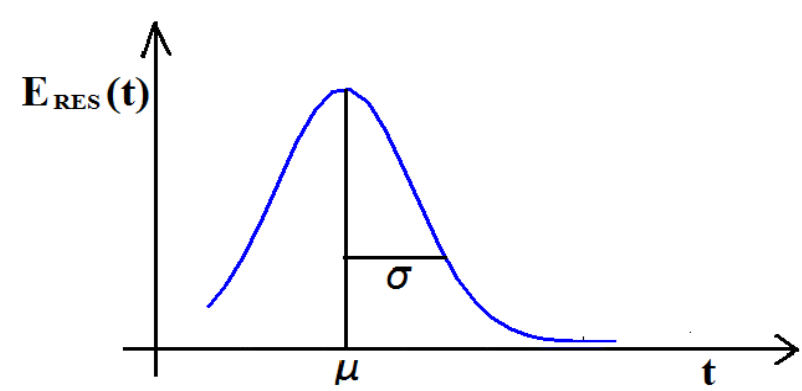

(a)

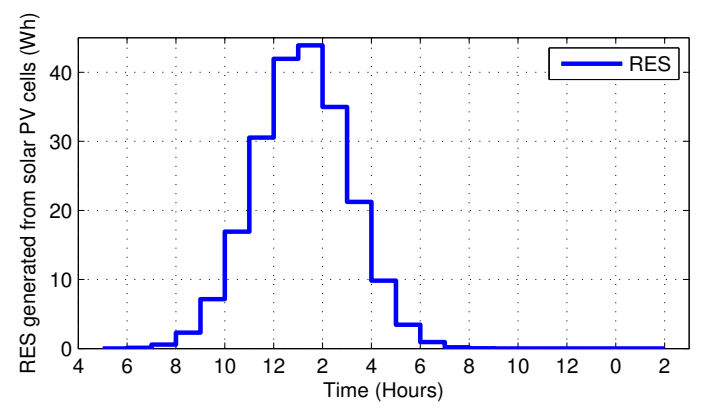

(b)

Figure 2. (a) Gaussian function representing the approximate PV cells' energy generation (Wh) [32]. (b) RES-generated energy $E_{R E S}$ for a single home.

The total daily energy generated from RESs must be positive, i.e., greater than zero and on a daily basis, it is given by:

$$
0 \leq E_{R E S} \leq E_{R E S_{(\max )}}
$$

where $E_{R E S_{(\max )}}$ is the maximum available RESs' generated energy capacity. If in any time interval, the RESs' generated energy exceeds the end-user energy demand $E_{T}$, i.e.,

$$
E_{R E S}>E_{T}
$$

then it is sold back to the grid as per their prior agreement or can be used for charging batteries in BSUs for later use, particularly during peak hours.

\subsection{BSU Model}

When RESs' generated energy exceeds the consumer energy demand, it is stored in the batteries using BSUs, which can be used during on-peak hours or night-time, when RESs are not available. This can be modelled using a binary variable $X_{\text {bat }}$ as:

$$
X_{b a t}= \begin{cases}1 & \text { for charging } \\ 0 & \text { for discharging }\end{cases}
$$

where $X_{b a t}$ shows the charging and discharging states of the batteries. In this model, we ignore the energy losses during the charging and discharging process. 


\subsection{Types of Users}

Residential users have been categorized as follows.

\subsubsection{Non-Active Users}

Non-Active Users (NAUs) do not use RESs and/or BSUs. They fulfil their load demand only from the utility grid. They can reduce their electricity bill by transferring their loads to off-peak hours. The consumed energy of NAU is calculated using the following equation:

$$
E_{N A U}=E_{T}
$$

where $E_{N A U}$ is the energy consumption of all non-active users and $E_{T}$ is the total energy consumption of all appliances.

\subsubsection{Semi-Active Users}

Semi-Active Users (SAUs) may generate their own energy using RESs and get energy from the grid when needed, i.e., when their demand exceeds the RESs' generated energy or when RESs are not available. That is, the end-user energy demand from the grid will be the total used energy minus their self-generated RES energy. Their consumed energy is calculated using the following equation:

$$
E_{S A U}=E_{T}-\sum_{n=1}^{48} E_{R E S, n}
$$

where $E_{S A U}$ is the energy consumption of semi-active users and $E_{R E S}$ is the energy generated from RESs.

\subsubsection{Fully-Active Users}

Fully-Active Users (FAUs) generate their own energy using RESs and store the extra generated energy in the batteries using BSUs. They obtain energy from the grid when needed, i.e., when their demand exceeds the RESs' generated energy plus the BSUs' stored energy. Their energy consumption pattern is calculated using the following equation:

$$
E_{F A U}=E_{T}-\sum_{n=1}^{48}\left(E_{R E S, n} \pm E_{B S U, n}\right)
$$

where $E_{F A U}$ is the energy consumption of fully-active users, $E_{R E S, n}$ is the $n$th consumer's RESs' generated energy and $E_{B S U, n}$ is consumers' stored energy using BSUs. Now, if $E_{B S U}$ is positive, this means that RESs are charging the batteries, and if $E_{B S U}$ is negative, this means batteries are discharging and providing energy to the load.

\section{Problem Formulation}

In this work, we assumed a single home and thirty homes with different power ratings of appliances, as tabulated in Table 2. Our required objectives are:

(a) Consumers' high comfort level by reducing appliances' average waiting time,

(b) Consumers' electricity bill minimization,

(c) Minimization of PAR and

(d) Integration of RES and BSU in the system for further reduction of end-user waiting time.

These objectives can be achieved by the optimization of the energy consumption profiles of home appliances, using different scheduling techniques. If $V_{T}$ is the maximum energy capacity in every time 
slot, then the end-user electricity cost along with PAR can be minimized, keeping aggregated energy consumption of cumulative home appliances within the maximum threshold limit of $V_{T}$.

Mathematically, this constraint can be shown as follows:

$$
E_{T} \leq V_{T}
$$

Here, $E_{T}$ is the total energy demand of the end-user.

Table 2. Appliances and their running time constraints [31]. LOT, Length of Operational Time.

\begin{tabular}{|c|c|c|c|c|c|c|c|}
\hline S. No. & Appliance & Category & $\begin{array}{l}\text { Power Rating } \\
\rho_{e, n}(\mathrm{KW})\end{array}$ & $\begin{array}{l}\text { Starting } \\
\text { Time }(\alpha)\end{array}$ & $\begin{array}{l}\text { Ending } \\
\text { Time }(\beta)\end{array}$ & $\begin{array}{l}\text { Time-Span } \\
(\beta-\alpha)(\mathrm{h})\end{array}$ & $\begin{array}{l}\text { LOT } \\
\text { (h) }\end{array}$ \\
\hline 1 & Fridge-1 & Fixed & 0.3 & 00 & 24 & 24 & 24 \\
\hline 2 & Interior Lighting-1 & Fixed & 0.84 & 18 & 24 & 06 & 6.0 \\
\hline 3 & Dish Washer-1 & Elastic & 2.0 & 09 & 17 & 08 & 2.0 \\
\hline 4 & Washing Machine-1 & Elastic & 0.6 & 09 & 12 & 03 & 1.5 \\
\hline 5 & Spin Dryer-1 & Elastic & 2.5 & 13 & 18 & 05 & 1.0 \\
\hline 6 & Cook Top-1 & Elastic & 3.0 & 08 & 09 & 01 & 0.5 \\
\hline 7 & Oven-1 & Elastic & 5.0 & 18 & 19 & 01 & 0.5 \\
\hline 8 & Microwave-1 & Elastic & 1.7 & 08 & 09 & 01 & 0.5 \\
\hline 9 & Laptop-1 & Elastic & 0.1 & 18 & 24 & 06 & 2.0 \\
\hline 10 & Desktop-1 & Elastic & 0.3 & 18 & 24 & 06 & 3.0 \\
\hline 11 & Vacuum Cleaner-1 & Elastic & 1.2 & 09 & 17 & 08 & 0.5 \\
\hline 12 & Electrical Car-1 & Elastic & 3.5 & 18 & 08 & 14 & 3.0 \\
\hline 1 & Fridge-2 & Fixed & 0.25 & 00 & 24 & 24 & 24 \\
\hline 2 & Interior Lighting-2 & Fixed & 0.9 & 19 & 24 & 07 & 7.0 \\
\hline 3 & Dish Washer-2 & Elastic & 1.9 & 11 & 15 & 04 & 2.0 \\
\hline 4 & Washing Machine-2 & Elastic & 0.5 & 10 & 14 & 04 & 2.0 \\
\hline 5 & Spin Dryer-2 & Elastic & 2.0 & 10 & 16 & 06 & 2.0 \\
\hline 6 & Cook Top-2 & Elastic & 3.5 & 09 & 10 & 01 & 0.5 \\
\hline 7 & Oven-2 & Elastic & 5.4 & 17 & 20 & 03 & 1.5 \\
\hline 8 & Microwave-2 & Elastic & 1.9 & 07 & 09 & 02 & 0.8 \\
\hline 9 & Laptop-2 & Elastic & 0.09 & 16 & 23 & 07 & 3.0 \\
\hline 10 & Desktop-2 & Elastic & 0.28 & 14 & 20 & 06 & 2.0 \\
\hline 11 & Vacuum Cleaner-2 & Elastic & 1.4 & 10 & 16 & 06 & 1.5 \\
\hline 12 & Electrical Car-2 & Elastic & 3.3 & 16 & 09 & 17 & 4.0 \\
\hline 1 & Fridge-3 & Fixed & 0.5 & 00 & 24 & 24 & 20 \\
\hline 2 & Interior Lighting-3 & Fixed & 0.62 & 17 & 06 & 13 & 13 \\
\hline 3 & Dish Washer-3 & Elastic & 2.5 & 10 & 16 & 06 & 2.5 \\
\hline 4 & Washing Machine-3 & Elastic & 0.8 & 08 & 14 & 06 & 1.8 \\
\hline 5 & Spin Dryer-3 & Elastic & 2.5 & 13 & 19 & 06 & 1.0 \\
\hline 6 & Cook Top-3 & Elastic & 3.2 & 07 & 09 & 02 & 0.5 \\
\hline 7 & Oven-3 & Elastic & 5.3 & 16 & 18 & 02 & 1.5 \\
\hline 8 & Microwave-3 & Elastic & 1.9 & 10 & 14 & 04 & 1.0 \\
\hline 9 & Laptop-3 & Elastic & 0.2 & 16 & 24 & 08 & 2.5 \\
\hline 10 & Desktop-3 & Elastic & 0.4 & 18 & 20 & 02 & 1.0 \\
\hline 11 & Vacuum Cleaner-3 & Elastic & 1.3 & 11 & 12 & 01 & 0.5 \\
\hline 12 & Electrical Car-3 & Elastic & 3.4 & 16 & 07 & 11 & 5.0 \\
\hline 1 & Fridge-4 & Fixed & 0.4 & 00 & 24 & 24 & 18 \\
\hline 2 & Interior Lighting-4 & Fixed & 0.7 & 19 & 08 & 13 & 13 \\
\hline 3 & Dish Washer-4 & Elastic & 2.3 & 08 & 19 & 11 & 4.0 \\
\hline 4 & Washing Machine-4 & Elastic & 0.9 & 11 & 14 & 03 & 1.0 \\
\hline 5 & Spin Dryer-4 & Elastic & 2.0 & 14 & 20 & 06 & 1.0 \\
\hline 6 & Cook Top-4 & Elastic & 3.5 & 10 & 12 & 02 & 1.2 \\
\hline 7 & Oven-4 & Elastic & 5.5 & 10 & 11 & 01 & 0.8 \\
\hline 8 & Microwave-4 & Elastic & 1.9 & 10 & 14 & 04 & 1.5 \\
\hline 9 & Laptop-4 & Elastic & 0.15 & 11 & 23 & 12 & 4.0 \\
\hline 10 & Desktop-4 & Elastic & 0.4 & 09 & 24 & 15 & 6.0 \\
\hline 11 & Vacuum Cleaner-4 & Elastic & 1.5 & 11 & 16 & 05 & 1.2 \\
\hline 12 & Electrical Car-4 & Elastic & 4.0 & 10 & 22 & 12 & 4.0 \\
\hline
\end{tabular}




\subsection{PAR}

The peak to average ratio can be minimized, using the scheduling algorithms, which is in favor of both the utility and consumer for maintaining demand-supply balance. It is the ratio of the peak load of the consumer to the average load of the consumer, in every interval of time, and is denoted by $\mu$. Mathematically, it is defined as in [33]:

$$
\mu=\frac{E_{\max }}{\frac{1}{T} \sum_{n=1}^{T} E_{T, n}}
$$

\subsection{User's Comfort in Terms of Waiting Time $\left(\tau_{w}\right)$}

User's comfort in terms of waiting time is important for end-users. Waiting time must be minimized to have a high comfort level so that the end-user's frustration can be avoided. It is that interval of time when a consumer switches on an appliance, and due to the scheduling limitations of the system, he/she has to wait to start its operation. As we have defined the earliest starting time $\alpha$ and the latest ending time $\beta$ of an appliance, then another parameter $\eta$ will be the operational starting time of the same switched-on appliance. This is shown diagrammatically in Figure 3. Here, $\beta-\alpha$ is the time span, defined by the consumer, as given in Table 2. Figure 3 shows that a consumer's maximum waiting time could be up to $\eta_{\max }$. Since LOT is already defined by the consumer, so at $\eta_{\max }$, the algorithm will have to start the appliance to complete its operation up to the final time $\beta$. For example, Dishwasher-1 (Table 2) has a time span of $8 \mathrm{~h}$ (from 9:00-17:00) and LOT $=2 \mathrm{~h}$. This means that our proposed algorithm (TG-MFO) must start Dishwasher-1 from 9:00-17:00 to complete its operation of $2 \mathrm{~h}$, with a waiting time ranging from $0-6 \mathrm{~h}$.

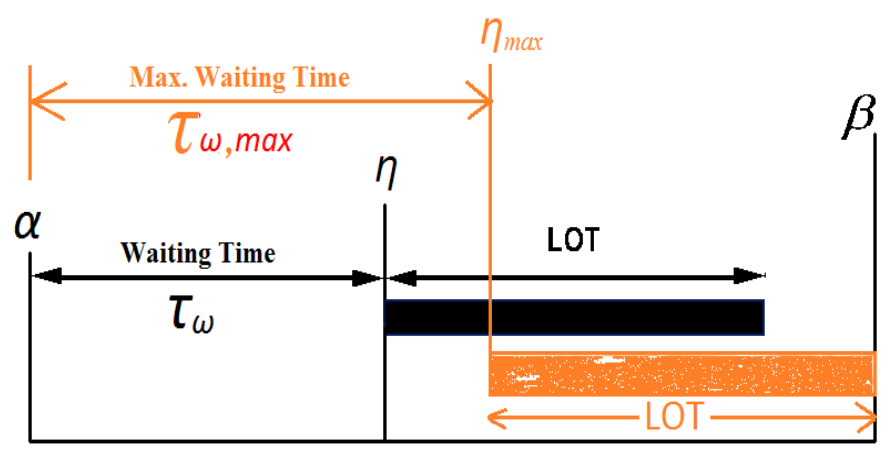

Figure 3. Starting time, ending time, LOT and waiting time.

Here, LOT is the length of the operational interval of time, in which an appliance completes its task. In the case of fixed appliances, there is no issue of waiting time, as whatever time the consumer wants to switch it on, he/she can do so. Therefore, we do not include fixed appliances in the scheduling problem.

Now, since,

$$
(\beta-\alpha) \geq L O T
$$

Therefore, the range of waiting time can be $\alpha$ to $\eta_{\max }$, as shown in Figure 3.

Appliances' normalized waiting time $\left(\tau_{w}\right)$ can be calculated as:

$$
\tau_{w}=\frac{\eta-\alpha}{(\beta-L O T)-\alpha}
$$

Equation (14) shows that the normalized waiting time can be from " 0 " (when $\eta=\alpha$ ) to " 1 " (when $(\beta-L O T)=\eta)$. Table 2 illustrates the typical electricity demand of a single home and multiple homes, with different power ratings and types of appliances, their LOTs and $(\alpha)$ and $(\beta)$ constraints [31]. Since 
different end-users have different habits and life routines, with different sizes and power ratings of appliances, we have assumed four (4) types of homes and randomly selected through the proposed algorithm, to have randomness in the consumed energy when taking multiple (here, 30 ) homes.

\subsection{Objective Function}

Mathematically, our objective function can be formulated as follows:

$$
\min \left(\sum_{m=1}^{48}\left[\lambda_{1} \times \sum_{n=1}^{N}\left(E_{T, n} \times \zeta_{n}\right)+\left(\lambda_{2} \times \tau_{w}\right)\right]\right)
$$

where $\zeta_{n}$ is the energy cost in every interval of time. Our proposed objective function aims to reduce electricity cost, while maintaining a higher end-user comfort level by minimization of waiting time. $\lambda_{1}$ and $\lambda_{2}$ are multiplying factors of two portions of the objective function. Their values can be either " 0 " or "1" so that $\left(\lambda_{1}+\lambda_{2}\right)=1$ [24]. This reveals that either $\lambda_{1}$ and $\lambda_{2}$ could be 0 or 1 . That is, if a consumer does not want to participate in the load scheduling process, then his/her multiplying factors will be $\lambda_{1}$ $=1$ and $\lambda_{2}=0$ in the objective function.

\section{Scheduling Algorithms}

Heuristic means "to discover", or "to find" or "to hit upon" by experiment, trial and error approaches. The solution of an optimization problem can be found in a realistic interval of time. However, such optimization techniques cannot guarantee the optimal solution. Meta-heuristic means "to find on a higher level" or "to find ahead of" local optimization. This means its performance is superior to straightforward heuristics techniques. All such algorithms use the process of local search and randomization, which further provides a path to global search and optimization.

The problem of scheduling home appliances optimally, using different meta-heuristic algorithms like GA, ACO, FA, MFO and CSA, is discussed in Section 6. Various classical programming techniques like LP, ILP, MILP, DP and CP have already been used by researchers for optimal scheduling of home appliances. The convergence time of these classical techniques is very large due to the exact solution, and to schedule a large number of appliances, they cannot be used. Furthermore, classical algorithms usually show the best results for local optimization, as compared to the global point of view. Therefore, due to their probabilistic nature, bio-inspired meta-heuristic algorithms give good results in the case of local, as well as global solutions.

\section{1. $G A$}

GA is in the family of evolutionary algorithms. This algorithm's name is due to it being inspired the genetic progression of living organisms. It has a quick rate of convergence. GA carries out parallel search operations in the provided solution space, which reduces the chances of being trapped in the local optimal solution. For complex non-linear problems' formulation, GA is the best option, especially where the global optimization is a challenging job. For any solution deployment, as GA is probabilistic in nature, the optimality is usually not guaranteed [34].

GA initiates a random population known as chromosomes, and then, in every iteration, the generated population is updated. Home appliances are mapped with bits of chromosomes. The fitness function of a given problem is evaluated by the suitability of each chromosome. In every iteration, the population is updated by storing the present local best solution, known as elitism. After elitism, in order to reproduce new chromosomes, two parent chromosomes are chosen using the tournament-based selection technique. Then, on the basis of selected chromosomes, the crossover procedure is performed. New offspring are added to update the present population [35]. Table 3 shows the GA parameters. 
Table 3. GA parameters.

\begin{tabular}{lll}
\hline S.No. & Parameter & Value \\
\hline 1 & Population size & 200 \\
2 & Crossover probability & 0.8 \\
3 & Mutation probability & 0.2 \\
4 & Maximum number of generations & 800 \\
\hline
\end{tabular}

\section{2. $M F O$}

The nature-inspired algorithm MFO was proposed by Seyedali Mirjalili in 2015 [36]. Moths are butterfly-like insects, having 160,000 plus different species in nature. They have their unique navigation mechanism known as transverse orientation when flying in the moonlight. When they fly in a spiral, they maintain a constant angle related to the moon, ultimately converging in the direction of light. The spiral articulates the searching region, and it assures the exploitation of the optimum solution.

Since MFO is a population-based algorithm, the movement of $m$ moths in $n$ dimensions (variables) is given in the position matrix form as follows:

$$
Q=\left[\begin{array}{ccc}
q_{1,1} & \ldots & q_{1, n} \\
\ldots & \ldots & \ldots \\
q_{m, 1} & \ldots & q_{m, n}
\end{array}\right]
$$

The resultant fitness values, for " $\mathrm{m}$ " number of moths, are stored in an array. The fitness function (objective) evaluates each moth's fitness value. Each moth's position vector, i.e., matrix Q's first row, is evaluated on the fitness function, and its output is then allocated to its respective moth.

Similarly, a matrix $U_{F}$ is assigned to the corresponding flames as follows:

$$
U_{f}=\left[\begin{array}{ccc}
u_{1,1} & \ldots & u_{1, n} \\
\ldots & \ldots & \ldots \\
u_{m, 1} & \ldots & u_{m, n}
\end{array}\right]
$$

Now, in mapping our problem of the optimum scheduling of home appliances, moths act as searching agents, and flames are the optimum positions. In each iteration, a moth searches for an optimum flame, with updates in the next iteration for the best solution by comparing with the previous one. Moths follow the logarithmic spiral for their update positions, where moths start from some initial position, following some limited fluctuating search space, and reach their destination flames. In MFO, the logarithmic spiral is:

$$
S\left(X_{i}, P_{j}\right)=d_{i} \cdot e^{b t} \cdot \cos (2 \pi t)+P_{j}
$$

where $d_{i}=\left|P_{j}-X_{i}\right|$ is the $i$ th moth distance from $j$ th flame, $b$ is the spiral shape defining the constant and the random number $t$ lies between -1 and one. When $t=-1$, this means the moth is closest to its destination flame, while $t=1$ indicates that its farthest position from the flame. Therefore, the moth is always assumed to be in a hyper-ellipse space, which guarantees the exploitation and exploration of search space. Table 4 depicts the MFO parameters.

Table 4. MFO parameters.

\begin{tabular}{lll}
\hline S. No. & Parameter & Value \\
\hline 1 & Number of moths and flames & 12 \\
2 & Max. No. of Iterations & 1000 \\
3 & Lower bound $L_{b}$ & -100 \\
4 & Upper bound $U_{b}$ & 100 \\
\hline
\end{tabular}




\subsection{TG-MFO}

In this work, we apply the bio-inspired algorithms GA, MFO and their hybrid version TG-MFO. Then, we compare their results with some of the existing techniques like ACO, CSA and FA on randomly-generated data. Then, we apply the strategy of the time constraints of end-users, i.e., for each appliance to switch-ON, we give some time span as the initial and final thresholds for all appliances. A user initiates the operation of an appliance, but usually, it is allowed to remain OFF for a certain time interval, in which the user has no problem or frustration. We apply this time threshold policy, to have a zero end-user waiting time. TG-MFO is based on the hybridization of GA and MFO with time constraints. Initially, MFO is applied on randomly-generated data for the optimization problem to obtain the local best positions for home appliances. Then, GA is applied to compare MFO's local best solution with the new random data, to find the global best solution in each iteration. The fitness functions are updated accordingly. This process continues until the termination criterion is fulfilled. Figure 4 depicts the steps involved in the TG-MFO implementation process, while, the pseudocode of Algorithm 1 shows the step-by-step working of the proposed TG-MFO algorithm.

\section{4. $A C O$}

ACO is a bio-inspired meta-heuristic iteration-based optimization technique. Using pheromone trails, chemicals as signals to other ants left on the ground known as the Stigmergy principle, starting from their nest, in search of food, ants find the shortest routes between their origins to the destination. If an ant wants, with a certain probability, to follow a particular path, it follows the pheromone trail. It reinforces the other ants by laying more pheromone on the same trail. As the movement of ants increases on a route, the amount of pheromone increases. Since pheromones' nature is volatile, so the shortest path has more pheromone as compared to the longer one. As a result, ants' movement increases on the shortest route.

Using this principle, ACO is used for the solution of discrete combinatorial search optimized-solution problems. Self-organization and self-healing are the distinguishing properties of ACO. ACO is used for the residential consumer's energy optimization, which is a novel scheme for such energy management problems [24]. The ACO parameters are given in Table 5.

Table 5. ACO parameters.

\begin{tabular}{llllll}
\hline S. No. & Parameter & Value & S. No. & Parameter & Value \\
\hline 1 & No. of Ants & 12 & 4 & Evaporation rate & 5 \\
2 & Pheromone intensity factor $\alpha_{1}$ & 2 & 5 & Trail decay factor & 0.5 \\
3 & Visibility intensity factor $\beta_{1}$ & 6 & 6 & Max. iterations & 600 \\
\hline
\end{tabular}




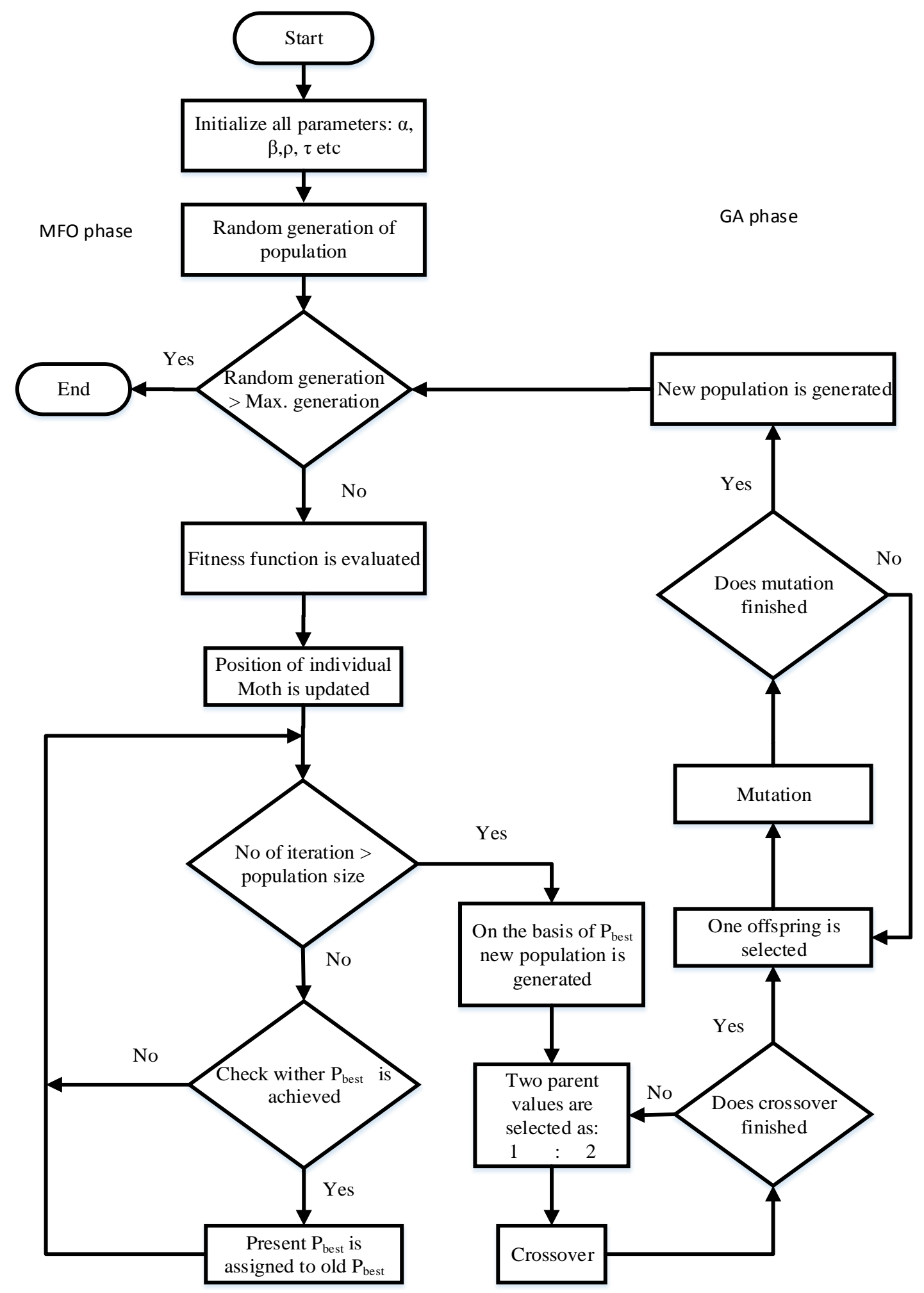

Figure 4. Flowchart for the proposed algorithm Time-constrained Genetic (TG)-MFO. 


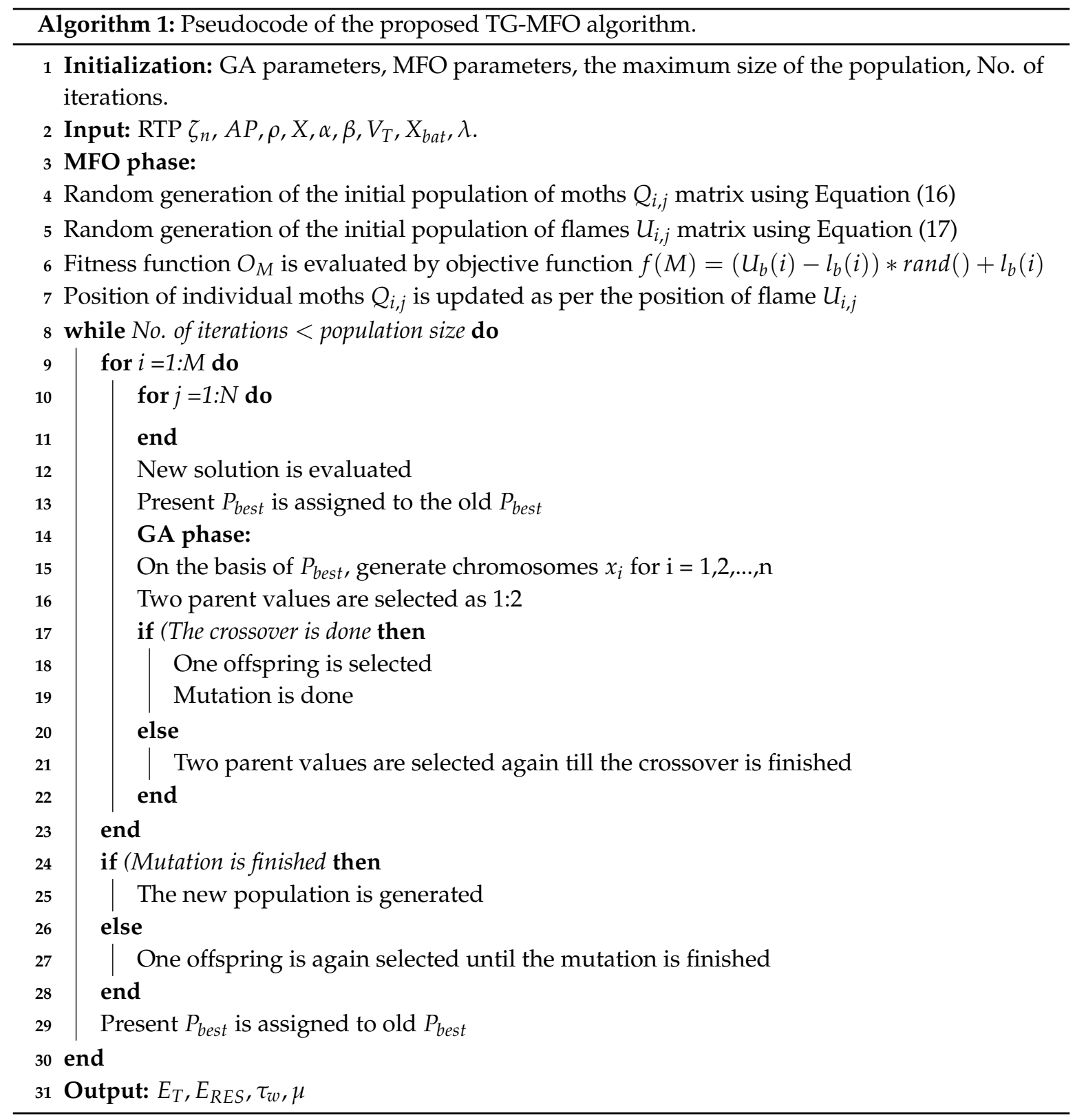

\section{5. $\operatorname{CSA}$}

The cuckoo search algorithm was proposed by [37] and belongs to the family of bio-inspired meta-heuristic algorithms. It is used to solve the optimization problems using the mating and production behaviour of some cuckoo species and the characteristics of Lèvy flights of some birds and fruit flies. Certain cuckoo species use nests of other birds to lay their eggs, selected randomly, known as host nests. The birds who own these nests may find these eggs and raise the cuckoo's young. CSA uses certain rules to find the best local solution by mapping pattern of eggs $(1,0)$ with home appliances' ON-OFF condition, stated as follows:

- The nest is randomly selected, in which each cuckoo lays one egg at a time.

- For upcoming production, those nests will be selected having the higher quality eggs.

- The host bird determines that the cuckoo laid the eggs, while the number of host nests is fixed.

The algorithm starts the discovery of the local best solution from randomly-given eggs (either one or zero) in the host nests. This one or zero shows the ON and OFF states of home appliances to 
be scheduled in a given time-slot. According to the fitness function, each egg (probable solution) is assessed. This solution will be our objective of minimum cost and PAR with reduced waiting time constrained. The production step is repeated, while discovering the superior eggs with a probability of 0.25 . Lèvy flights are carried out to find the global best solution, out of the local best solution [38]. Table 6 gives the CSA parameters.

Table 6. CSA parameters.

\begin{tabular}{lll}
\hline S. No. & Parameter & Value \\
\hline 1 & Number of host nests & 12 \\
2 & Number of iterations & 1000 \\
3 & Discovery rate & -100 \\
\hline
\end{tabular}

\section{6. $F A$}

Fireflies are in the insect family. They live mostly in humid environments. They generate green, yellow and pale-red limited intensity flashing lights chemically. There are more than 2000 different species. Their unique flashing light pattern is used for communication, i.e., to attract partners and probable prey and as a defensive cautionary mechanism. Some female species apply the flashing light mating pattern for the hunting of other species [39].

Like PSO, in FA, inspired by nature, three assumptions are made: (a) all fireflies must be of the same sex, (b) the attractiveness of a firefly is directly proportional to its brightness and inversely proportional to the square of the distance between two fireflies and (c) brightness is calculated by an objective function: the brighter one will attract the less bright ones. The firefly's flashing light intensity in complete darkness is related to the solution quality. The brighter firefly will attract less bright ones, depending on the brightness intensity, which is calculated as follows:

$$
I\left(I_{0}, r_{i, j}\right)=\frac{I_{0}}{r_{i, j}^{2}}
$$

where $I_{o}$ is the flashing light intensity at the origin and $r_{i, j}$ is the distance of firefly $\mathrm{j}$ from firefly $\mathrm{i}$. Let $\gamma$ be the coefficient of the firefly's flashing light absorption in a medium, then, in the above equation, the light intensity I will vary with the distance between fireflies $r_{i, j}$ using the following equation:

$$
I\left(I_{0}, \gamma, r_{i, j}\right)=I_{0} e^{-\gamma r_{i, j}}
$$

Both Equations (19) and (20) can be combined using the Gaussian form as follows:

$$
I\left(I_{0}, \gamma, r_{i, j}\right)=I_{0} e^{-\gamma r_{i, j}^{2}}
$$

The following approximation can be used for a slower rate of decrease in the light intensity between the origin and the target.

$$
I\left(I_{o}, \gamma, r_{i, j}\right)=\frac{I_{0}}{1+\gamma r_{i, j}^{2}}
$$

As the less bright firefly will be attracted to the brighter firefly, this attractiveness $\beta$ between two fireflies can now be mapped as:

$$
\beta\left(\beta_{0}, \gamma, r_{i, j}\right)=\beta_{o} e^{-\gamma r_{i, j}^{2}}
$$


where $\beta_{o}$ is the attractiveness at the zero distance. For $\beta=\beta_{o}$ at the zero distance, $r_{i, j}=0$. Therefore, for a characteristic distance of $r=\frac{1}{\sqrt{\gamma}}$, Equation (23) becomes: $\beta\left(\beta_{o}, \gamma, r_{i, j}\right)=\beta_{o} e^{-1}$. The distance between any two fireflies $\mathrm{x}$ and $\mathrm{y}$ at positions $i$ and $j$ is calculated by:

$$
r_{i, j}=\operatorname{Dist} .\left(x_{i}, y_{j}\right)=\left\|x_{i}-y_{j}\right\|=\sqrt{\sum_{k=1}^{n}\left(x_{i, k}-y_{j, k}\right)^{2}}
$$

The firefly movement towards another firefly has two parts:

(a) The movement will be for finding a better solution using attractiveness.

(b) The movement will be random.

$$
\begin{gathered}
x_{i}=x_{i}+(\text { Attractiveness } * \text { Distance })+\text { Randomness } \\
x_{i}=x_{i}+\beta_{o} e^{-\gamma r_{i, j}^{2}} \cdot\left(y_{j}-x_{i}\right)+\alpha(\operatorname{Rand}()-0.5)
\end{gathered}
$$

where $\alpha$ is the randomness parameter and Rand is a random number generated lying between zero and one. Two extreme points are that when $\gamma$ is zero, attractiveness will be constant, and when $\gamma$ is $\infty$, attractiveness will almost be zero. Practically, $\gamma$ lies between zero and $\infty$, so FA gives good results in finding local, as well as global optima [39]. Table 7 depicts the FA parameters.

Table 7. FA parameters.

\begin{tabular}{lll}
\hline S. No. & Parameter & Value \\
\hline 1 & Randomness parameter $(\alpha)$ & 0.2 \\
2 & Attractiveness $(\beta)$ & 2 \\
3 & Absorption coefficient $(\gamma)$ & 1 \\
\hline
\end{tabular}

\section{Results and Discussions}

\subsection{Consumer Scenarios}

In the present work, four types of consumer scenarios were simulated and discussed. In the first case, a single home was taken, and its hourly load, hourly energy cost, PAR and waiting time were determined both in the unscheduled and scheduled (with ACO, CSA, GA, FA, MFO and TG-MFO) environment for a single day. In the second case, a residential building with thirty homes having users with different habits with different LOTs and different power ratings of appliances were considered. Again, their hourly average load, hourly cost, PAR and waiting time were determined both for unscheduled and scheduled (with ACO, CSA, GA, FA, MFO and TG-MFO) scenarios for a single day. In the third case, we considered a single home, found all four of its parameters for unscheduled and scheduled scenarios for a complete month, i.e., thirty (30) days, and found its monthly bill. In the fourth case, a residential building with thirty homes was considered, and we determined its daily average load, daily cost, daily PAR and average waiting time for a complete month, i.e., thirty (30) days, as well as calculated its monthly electricity consumption and electricity bill. In all four cases, the RTP scheme was used.

For the system's stability, further reduction of electricity consumption and maximum user comfort, RES and BSUs were also integrated. Additionally, for photovoltaic cells' electricity generation, temperature, solar irradiance, battery charging/discharging rates and its storage system, different assumptions were considered from [40].

\subsection{Pricing Signal}

Different pricing signals were available. We used the day-ahead real time pricing (RTP) signal in our simulations, as shown in Figure 5. 


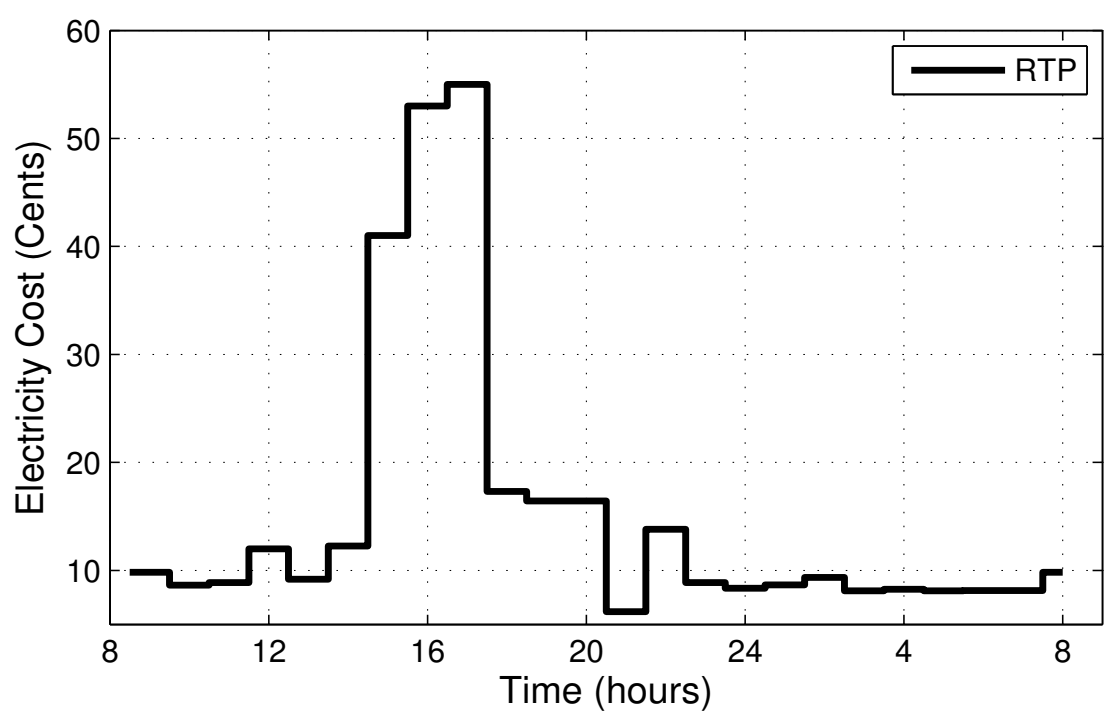

Figure 5. Day-ahead real-time pricing signal.

\subsection{The Average Waiting Time}

Waiting time is a very important feature of appliance scheduling for optimal energy consumption in the smart grid. To reduce electricity cost, usually, waiting time increases. A user wants to start an appliance, but due to scheduling time constraints, the user has to wait for to start its operation. Our main objective in this work was to minimize the user electricity bill, keeping in view the maximum comfort level of the end-user. Figure 6 shows that we achieved our objective using heuristic techniques for optimal scheduling. The graphs show that TG-MFO outperformed ACO, CSA, GA, FA and MFO in achieving a nearly-zero waiting time for the end-user.

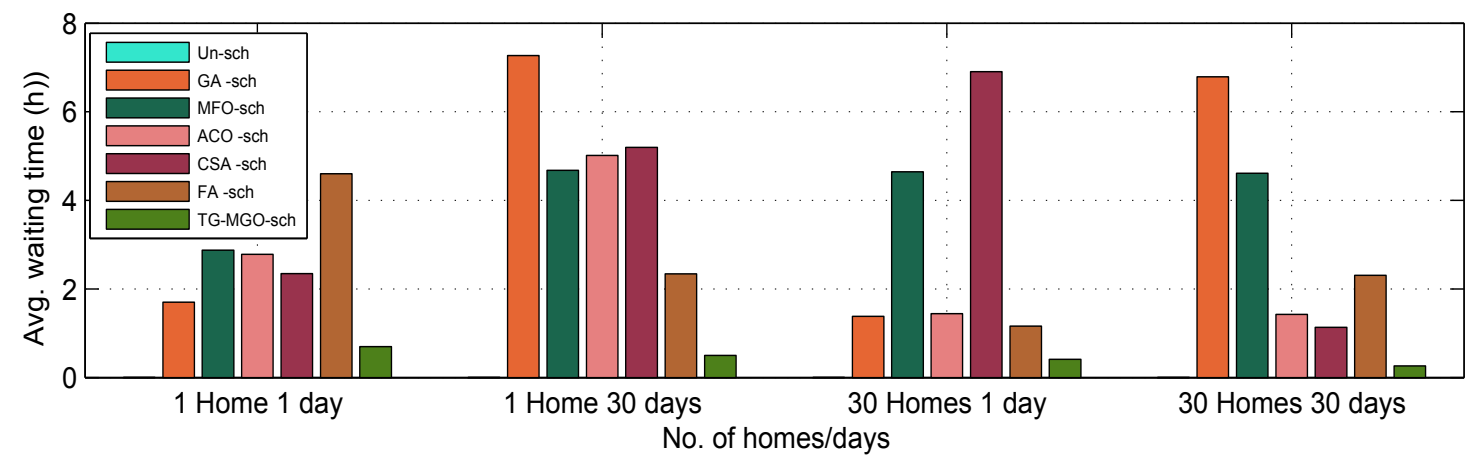

Figure 6. Average waiting time for a single home and 30 homes.

\subsection{The Total Electricity Cost}

Figure 7 shows the electricity cost for the unscheduled and scheduled (with ACO, CSA, GA, FA, MFO and TG-MFO) load. The results show that the electricity cost of the meta-heuristic algorithms-based scheduled load was very low as compared to the unscheduled load cost. In Figure 7a, the operation of a single home for a single day is considered. In this case, ACO-based scheduling showed better results as compared to all scheduled and unscheduled costs. Similarly, in Figure $7 \mathrm{~b}$, the case of a single home for 30 days (one month) is shown, in Figure 7c, that of 30 homes with different LOTs and power ratings for 30 days (one month), and in Figure $7 \mathrm{~d}$, the case of 30 homes for 30 days; the scheduled cost was very much as compared to the un-scheduled cost. In all four cases, ACO outperformed all scheduling techniques. 


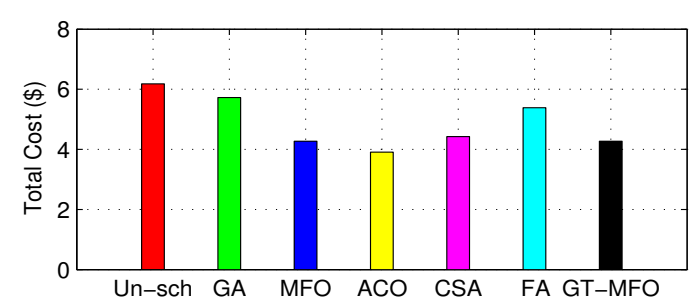

(a)

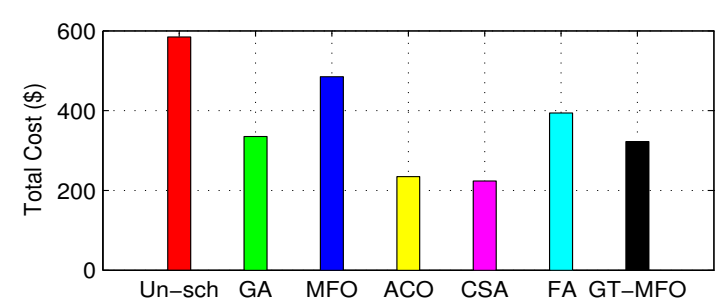

(c)

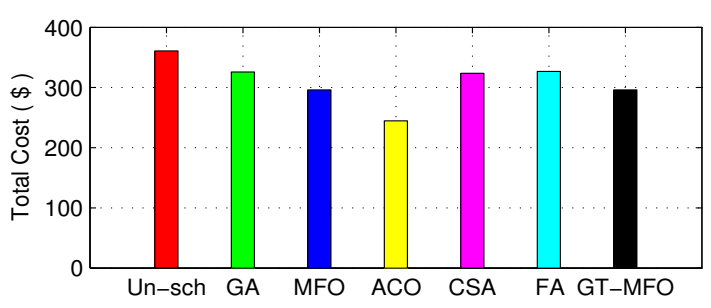

(b)

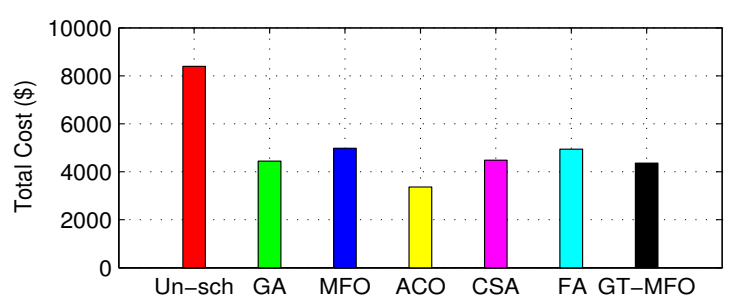

(d)

Figure 7. Total cost for un-scheduled, and GA, MFO, ACO, CSA, FA and TG-MFO Scheduled load (a) Total cost for a single home for 1 day. (b) Total cost for single home for 30 days. (c) Total cost for 30 homes for 1 day. (d)Total cost for 30 homes for 30 days.

\subsection{Hourly Load}

Figure 8 shows the total hourly load of a single home and 30 homes with unscheduled and scheduled loads with the bio-inspired algorithms ACO, CSA, GA, FA, MFO and TG-MFO. The results show that as compared to the unscheduled load, meta-heuristic algorithm-based scheduled load was shifted to the off-peak hours, where not only the price was low, but RES was also available, considering the end-user's time constraints for the maximum comfort level. The figure shows that our proposed algorithm gave comparative results for both single and multiple homes with different sizes, power ratings and LOTs.

\subsection{Integration of RES and BSU}

In order to minimize the consumed energy for further reduction of the total cost, RES and BSUs were integrated in homes. Figure 9 shows that the day-time load will be supported by RES, while extra energy will be stored in BSUs for running the load in peak hours. It drastically reduced the cost. The figure depicts that our proposed TG-MFO algorithm has intelligently not only shifted the load to day-time off-peak hours for cost minimization, but also reduced the PAR.

\subsection{PAR}

PAR plays an important role in the optimal scheduling of smart home appliances. Due to high PAR, the utility faces huge peak loads during peak hours, and the rest of the day, most of the generating units remain idle. Therefore, researchers try to reduce PAR for economical load dispatch in smart grids. Figure 10 shows that, in the case of a single home for a single day and thirty homes for a single day, MFO performed better than FA, while in the case of a single home for thirty days and thirty homes for thirty days, FA showed better results than MFO. In our proposed hybrid model, we tried to not only schedule appliances optimally, economically and having maximum end-user comfort, but also gave the lowest PAR, for the benefit of utility, and hence, to further increase the end-user comfort level. 


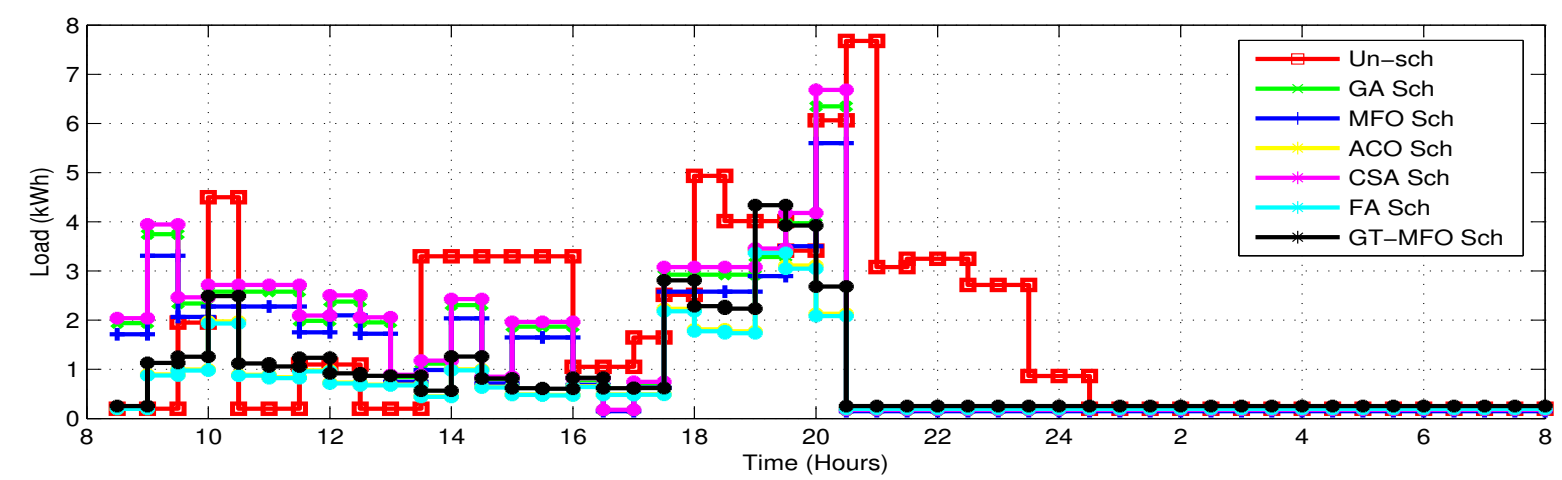

(a)

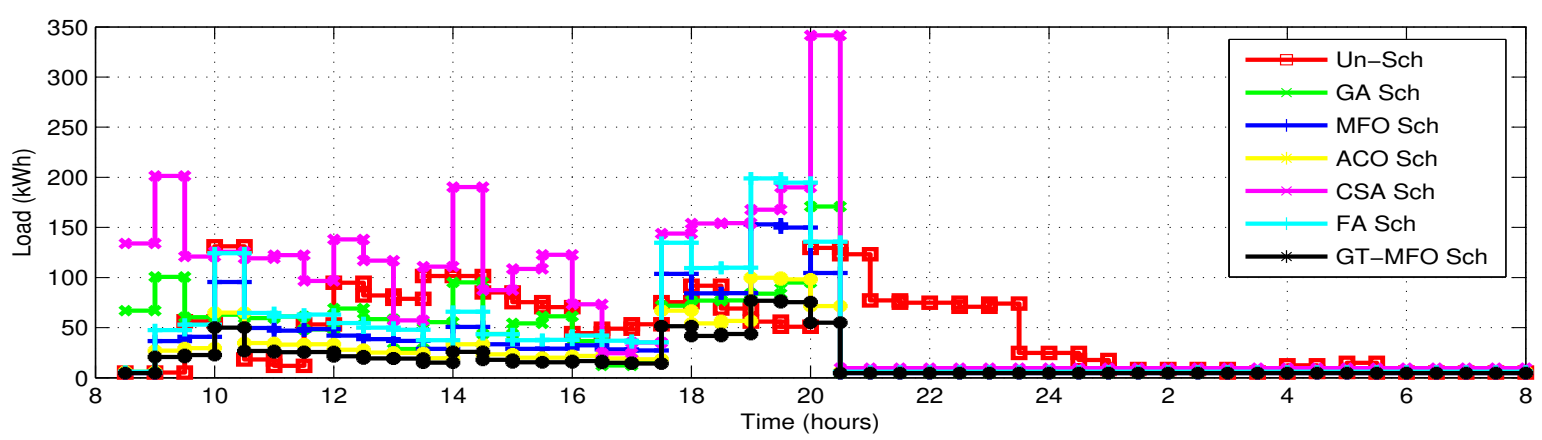

(b)

Figure 8. The hourly load for un-scheduled and GA-, MFO-, ACO-, CSA-, FA- and TG-MFO-scheduled load. (a) The hourly load for a single home. (b) The hourly load for 30 homes.

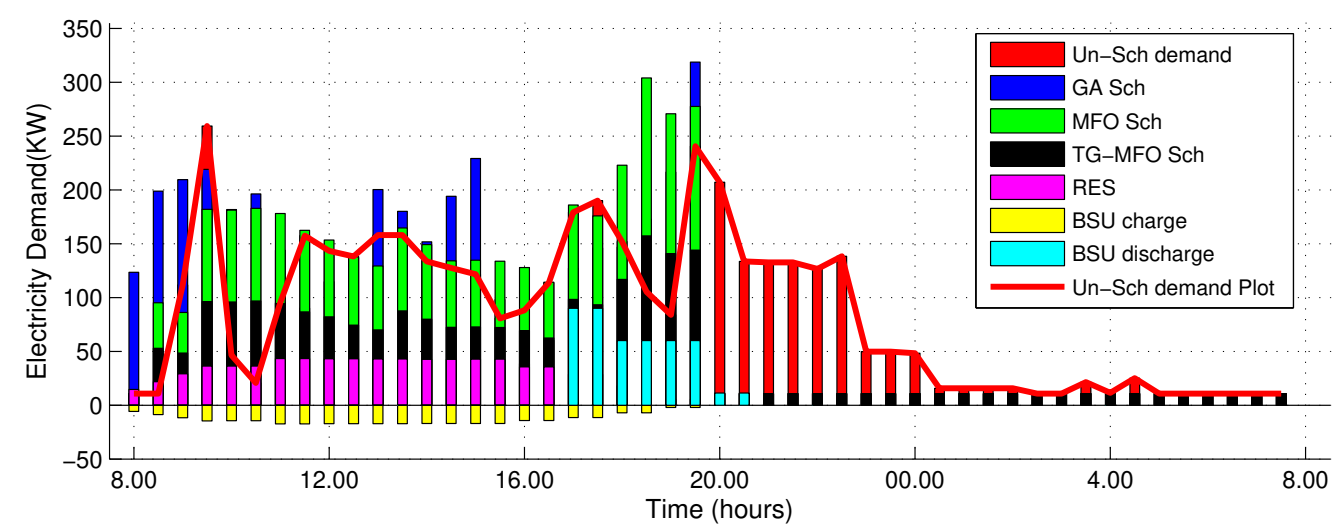

Figure 9. Energy demand curves of the scheduled and the un-scheduled load along with RES and BSUs.

A comparison of the proposed algorithm with state-of-the-art research works in the smart grid environment for energy optimization and end-user comfort is depicted in Table 8. Most of the existing techniques have used trade-offs between user comfort and bill minimization. 


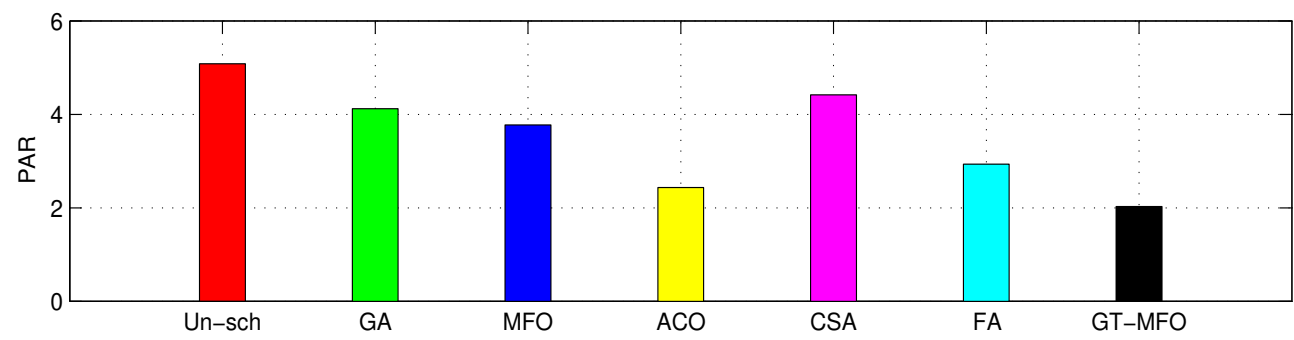

(a)

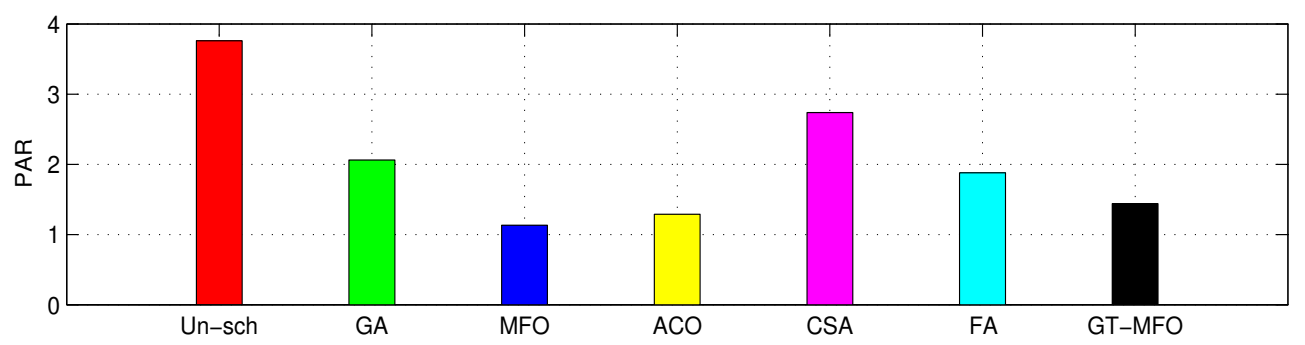

(b)

Figure 10. PAR for un-scheduled and GA-, MFO-, ACO-, CSA-, FA- and TG-MFO-scheduled load. (a) PAR for a single home. (b) PAR for 30 homes.

Table 8. Comparison of TG-MFO with the state-of-the-art work.

\begin{tabular}{llll}
\hline Techniques & No. of Homes/Days & $\begin{array}{l}\text { Cost } \\
\text { Reduction }\end{array}$ & Waiting Time \\
\hline K. Ma et al. [5] & $34 \%$ & $1.76 \mathrm{~h}$ \\
\hline Y. Peizhong et al. [8] & 30 days & $20 \%$ & $7.64 \mathrm{~h}$ \\
\hline TLGO [14] & & $33 \%$ & $1.83 \mathrm{~h}$ \\
TLBO & $31.5 \%$ & $2.14 \mathrm{~h}$ \\
GA & $31 \%$ & $2.37 \mathrm{~h}$ \\
\hline Ogunjuyigbe et al. [23] & & 9.6 (dis-satisfaction level) \\
& & & $20.2=$ \\
& & $30.9=$ \\
\hline Pilloni et al. [25] & 10 Home appliances & $38 \%$ & $1.65 \mathrm{to} 1.70$ (Annoyance rate) \\
\hline K. Muralitharan et al. [28] & & $73.32 \mathrm{~s}$ \\
& 11 & & $76.28 \mathrm{~s}$ \\
& 12 & $96.83 \mathrm{~s}$ \\
& 13 & $107.58 \mathrm{~s}$ \\
\hline TG-MFO (Proposed) & 14 & $32.25 \%$ & $0.62 \mathrm{~h}$ \\
& 1 home for 1 day & $0.48 \mathrm{~h}$ \\
& 1 home for 30 days & $19.39 \%$ & $0.38 \mathrm{~h}$ \\
& 30 homes for 1 day & $43.98 \%$ & $0.26 \mathrm{~h}$ \\
\hline
\end{tabular}

Table 9 shows the performance of the proposed TG-MFO algorithm, compared to unscheduled load and scheduled with the GA, MFO, ACO, FA and CSA algorithms. 
Table 9. Comparison of the un-scheduled load with GA, MFO, ACO, CSA, FA and TG-MFO.

\begin{tabular}{|c|c|c|c|c|c|c|}
\hline Techniques & No. of Homes/Days & Cost (\$) & $\begin{array}{l}\text { \%Cost } \\
\text { Reduction }\end{array}$ & $\begin{array}{l}\text { Waiting } \\
\text { Time (h) }\end{array}$ & PAR & $\begin{array}{l}\text { \% PAR } \\
\text { Change }\end{array}$ \\
\hline Un- & Single home for 1 day & 6.2 & - & - & 4.58 & - \\
\hline \multirow{3}{*}{ Schedule } & Single home for 30 days & 366 & - & - & - & - \\
\hline & 30 homes for 1 day & 582 & - & - & 3.84 & - \\
\hline & 30 homes for 30 days & 8476 & - & - & - & - \\
\hline GA & Single home for 1 day & 5.8 & $06.45 \%$ & 1.75 & 4.19 & $8.5 \%$ \\
\hline \multirow[t]{3}{*}{ Scheduled } & Single home for 30 days & 332 & $09.28 \%$ & 7.41 & - & - \\
\hline & 30 homes for 1 day & 340 & $41.58 \%$ & 1.45 & 2.13 & $44.5 \%$ \\
\hline & 30 homes for 30 days & 4320 & $49.03 \%$ & 6.76 & - & - \\
\hline $\mathrm{MFO}$ & Single home for 1 day & 4.2 & $32.26 \%$ & 2.81 & 3.82 & $16.6 \%$ \\
\hline \multirow[t]{3}{*}{ Scheduled } & single home for 30 days & 296 & $19.12 \%$ & 4.62 & - & - \\
\hline & 30 homes for 1 day & 490 & $15.8 \%$ & 4.67 & 1.21 & $68.5 \%$ \\
\hline & 30 homes for 30 days & 4992 & $41.10 \%$ & 4.61 & - & - \\
\hline TG-MFO & Single home for 1 day & 4.2 & $32.25 \%$ & 0.62 & 2.02 & $49.8 \%$ \\
\hline \multirow[t]{3}{*}{ Scheduled } & single home for 30 days & 295 & $19.39 \%$ & 0.48 & - & - \\
\hline & 30 homes for 1 day & 326 & $43.98 \%$ & 0.38 & 1.48 & $61.4 \%$ \\
\hline & 30 homes for 30 days & 4241 & $49.96 \%$ & 0.26 & - & - \\
\hline $\mathrm{ACO}$ & Single home for 1 day & 4.4 & $29.03 \%$ & 2.74 & 2.34 & $48.9 \%$ \\
\hline \multirow[t]{3}{*}{ Scheduled } & Single home for 30 days & 245 & $33.06 \%$ & 5.02 & - & - \\
\hline & 30 homes for 1 day & 242 & $58.41 \%$ & 1.48 & 1.37 & $64.3 \%$ \\
\hline & 30 homes for 30 days & 3212 & $62.10 \%$ & 1.39 & - & - \\
\hline CSA & Single home for 1 day & 4.38 & $29.35 \%$ & 2.46 & 4.26 & $6.9 \%$ \\
\hline \multirow[t]{3}{*}{ Scheduled } & Single home for 30 days & 336 & $08.19 \%$ & 5.21 & - & - \\
\hline & 30 homes for 1 day & 239 & $58.93 \%$ & 6.82 & 2.67 & $30.4 \%$ \\
\hline & 30 homes for 30 days & 4295 & $49.32 \%$ & 1.18 & - & - \\
\hline FA & Single home for 1 day & 5.2 & $16.12 \%$ & 4.54 & 2.45 & $46.5 \%$ \\
\hline \multirow[t]{3}{*}{ Scheduled } & Single home for 30 days & 338 & $07.65 \%$ & 2.38 & - & - \\
\hline & 30 homes for 1 day & 398 & $31.61 \%$ & 1.17 & 1.83 & $52.3 \%$ \\
\hline & 30 homes for 30 days & 4852 & $42.75 \%$ & 2.32 & - & - \\
\hline
\end{tabular}

Table 10 shows the runtime of the proposed algorithms using an Intel (R) Core (TM) i5 processor, with $4.00 \mathrm{~GB}$ of installed memory (RAM) and the 32-bit Windows 7 Operating system.

Table 10. Runtime of the proposed algorithm in four different scenarios.

\begin{tabular}{lll}
\hline Proposed Algorithm & No. of Homes/Days & Run Time (s) \\
\hline TG-MFO & Single home for 1 day & 47.65 \\
& Single home for 30 days & 123.02 \\
& Thirty homes for 1 day & 129.18 \\
& Thirty homes for 30 days & 841.65 \\
\hline
\end{tabular}

\section{Conclusions and Future Work}

In this paper, we mapped GA, MFO and a new efficient and robust hybrid TG-MFO meta-heuristic bio-inspired algorithm for optimal scheduling of home appliances in the smart grid and compared their results with existing techniques of CSA, FA and ACO. We considered a single- and multiple home scenarios in a residential sector. In multiple homes, we took different LOTs and power ratings of appliances to make it more practical. Day-ahead RTP signalling was used for demand response in smart homes. The results show that there was a $6.45 \%-49.03 \%, 32.26 \%-41.10 \%$ and $32.25 \%-49.96 \%$ decrease in the total cost with GA, MFO and TG-MFO scheduling, respectively, for single and multiple users. RESs and BSUs were also integrated to obtain a further decrease in the total cost and end-user 
waiting time. In this work, we tried to not only reduce the total cost, but to achieve a high comfort level of the end-user by minimizing the waiting time of home appliances using the time constraints of a maximum average delay of $0.26-0.62 \mathrm{~h}$. This algorithm can be applied to actual data when and where they are provided. It not only reduces the energy cost, but also increases the stability and reliability of the grid. Future work includes exploration of more bio-inspired algorithms for intelligent and efficient energy optimization, and a multi-objective approach will be applied.

Author Contributions: I.U. wrote the first draft of the manuscript, proposed the idea and topology design method. S.H. provided technical supervision, insights and additional ideas on presentation. Both of the authors equally contributed, revised and approved the manuscript.

Funding: This research received no external funding.

Conflicts of Interest: The authors declare no conflict of interest.

\section{References}

1. Sesana, M.M.; Salvalai, G. Overview on life cycle methodologies and economic feasibility for nZEBs. Build. Environ. 2013, 67, 211-216. [CrossRef]

2. Lo, C.H.; Ansari, N. The progressive smart grid system from both power and communications aspects. IEEE Commun. Surv. Tutor 2012, 14, 799-821. [CrossRef]

3. Javeed, F.; Arshad, N. On the Use of Linear Programming in Optimizing Energy Costs. In Procedings of the International Workshop on Self-Organizing Systems, Vienna, Austria, 10-12 December 2008; pp. 305-310.

4. Huang, Y.; Tian, H.; Wang, L. Demand response for home energy management system. Int. J. Electr. Power Energy Syst. 2015, 73, 448-455. [CrossRef]

5. Ma, K.; Yao, T.; Yang, Y.; Guan, X. Residential power scheduling for demand response in smart grid. Int. J. Electr. Power Energy Syst. 2016, 78, 320-325. [CrossRef]

6. Naraharisetti, P.K.; Karimi, I.A.; Anand, A.; Lee, D. A linear diversity constraint application to scheduling in microgrids. Energy 2011, 36, 4235-4243. [CrossRef]

7. Fuselli, D.; de Angelis, F.; Boaro, M.; Squartini, S.; Wei, Q. Action dependent heuristic dynamic programming for home energy resource scheduling. Int. J. Elect. Power Energy Syst. 2013, 48, 148-160. [CrossRef]

8. Yi, P.; Dong, X.; Iwayemi, A.; Zhou, C.; Li, S. Real-time opportunistic scheduling for residential demand response. IEEE Trans. Smart Grid 2013, 4, 227-234. [CrossRef]

9. Sianaki, O.A.; Hussian, O.; Tabesh, A.R. A Knapsack Problem Approach for Achieving Efficient Energy Consumption in Smart Grid for End-user Life Style. In Proceedings of the IEEE Conference on Innovative Technologies for an Efficient and Reliable Electricity Supply Waltham, MA, USA, 27-29 September 2010; pp. 159-164.

10. Javaid, N.; Hussain, S.M.; Ullah, I.; Noor, M.A.; Abdul, W. Demand Side Management in Nearly Zero Energy Buildings Using Heuristic Optimizations. Energies 2017, 10, 1131-1159. [CrossRef]

11. Kumaraguruparan, N.; Sivaramakrishnan, H.; Sapatnekar, S. Residential task scheduling under dynamic pricing using the multiple knapsack method. In Proceedings of the International Conference on, Innovative Smart Grid Technologies, Washington, DC, USA, 16-20 January 2012; pp. 1-6.

12. Huang, Y.; Wang, L.; Guo, W.; Kang, Q.; Wu, Q. Chance Constrained Optimization in a Home Energy Management System. IEEE Trans. Smart Grid 2018, 9, 252-260. [CrossRef]

13. Javaid, N.; Ahmed, F.; Ullah, I. Towards Cost and Comfort Based Hybrid Optimization for Residential Load Scheduling in a Smart Grid. Energies 2017, 10, 1546. [CrossRef]

14. Manzoor, A.; Javaid, N.; Ullah, I.; Abdul, W. An Intelligent Hybrid Heuristic Scheme for Smart Metering based Demand Side Management in Smart Homes. Energies 2017, 10, 1258. [CrossRef]

15. Ma, K.; Shubing, H.; Jie, Y.; Xia, X.; Xinping, G. Appliances scheduling via cooperative multi-swarm PSO under day-ahead prices and photovoltaic generation. Appl. Soft Comput. 2018, 62, 504-513. [CrossRef]

16. Khuri, S.; Back, T.; Heitkotter, J. The Zero/One Multiple Knapsack Problem and GA. In Proceedings of the 1994 ACM Symposium on Applied Computing, Phoenix, AZ, USA, 6-8 March 2011; pp. 188-193.

17. Ogwumike, C.; Short, M.; Denai, M. Near-Optimal Scheduling of Residential SH Appliances using Heuristic Approach. In Proceedings of the 2015 IEEE International Conference on Industrial Technolog, Seville, Spain, 17-19 March 2015; pp. 3128-3133. 
18. Kusakana, K. Energy management of a grid-connected hydrokinetic system under ToU tariff. Energy 2017, 101, 1325-1333.

19. Bharathi, C.; Rekha, D.; Vijayakumar, V. Genetic Algorithm Based Demand Side Management for Smart Grid. Wirel. Pers. Commun. 2017, 93, 481-502. [CrossRef]

20. Muratori, M., Rizzoni, G. Residential demand response: dynamic energy management and time-varying electricity pricing. IEEE Trans. Power Syst. 2016, 31, 1108-1117. [CrossRef]

21. Ogunjuyigbe, A.S.O.; Ayodele, T.R.; Akinola, O.A. User satisfaction-induced demand side load management in residential buildings with user budget constraint. Appl. Energy 2017, 187, 352-366. [CrossRef]

22. Logenthiran, T.; Srinivasan, D.; Shun, T.Z. Demand Side Management in Smart Grid Using Heuristic Optimization. IEEE Trans. Smart Grid 2012, 3, 1244-1252. [CrossRef]

23. Pilloni, V.; Floris, A.; Meloni, A.; Atzori, L. Smart Home Energy Management Including Renewable Sources: A QoE-driven Approach. IEEE Trans. Smart Grid 2018., 2016. [CrossRef]

24. Rahim, S.; Javaid, N.; Ahmad, A. Exploiting heuristic algorithms to efficiently utilize energy management controllers with renewable energy sources. Energy Build. 2016, 129, 452-470. [CrossRef]

25. Ahmad, A.; Khan, A.; Javaid, N. An Optimized Home Energy Management System with Integrated Renewable Energy and Storage Resources. Energies 2017, 10, 549. [CrossRef]

26. Muralitharan, K.; Sakthivel, R.; Shi, Y. Multiobjective optimization technique for demand side management with load balancing approach in smart grid. Neurocomputing 2016, 177, 110-119. [CrossRef]

27. Moon, S., Lee, J.W. Multi-Residential Demand Response Scheduling with Multi-Class Appliances in Smart Grid. IEEE Trans. Smart Grid 2018, 9, 2518-2528. [CrossRef]

28. Li, C.; Yu, X.; Yu, W.; Chen, G.; Wang, J. Efficient computation for sparse load shifting in demand side management. IEEE Trans. Smart Grid 2017, 8, 250-261. [CrossRef]

29. Albu, M.M.; Snduleac, M.; Stnescu, C. Syncretic use of smart meters for power quality monitoring in emerging networks. IEEE Trans. Smart Grid 2017, 8, 485-492. [CrossRef]

30. Saxena, N.; Grijalva, S. Dynamic secrets and secret keys based scheme for securing last mile smart grid wireless communication. IEEE Trans. Ind. Inform. 2017, 13, 1482-1491. [CrossRef]

31. Zhang, D.; Evangelisti, S.; Lettieri, P.; Lazaros, G. Economic and environmental scheduling of smart homes with microgrid: DER operation and electrical tasks. Energy Convers. Manag. 2015, 89, 228-249. [CrossRef]

32. Monteiro, C.; Santos, T.; Jimenez, L.A.F.; Rosado, I.J.R.; Olarte, M.S.T. Short-Term Power Forecasting Model for Photovoltaic Plants based on Historical Similarity. Energies 2013, 6, 2624-2643. [CrossRef]

33. Liu, Y.; Yuen, C.; Huang, S. Peak-to-Average Ratio Constrained Demand-Side Management With Consumer's Preference in Residential Smart Grid. IEEE J. Sel. Top. Signal Proc. 2014, 8, 1084-1097. [CrossRef]

34. Mardle, S.; Pascoe, S. An overview of Genetic Algorithms for the Solution of Optimization Problems. CHEER 1999, 13, 16-20.

35. Aslam, S.; Iqbal, Z.; Javaid, N. Towards Efficient Energy Management of Smart Buildings Exploiting Heuristic Optimization with Real Time and Critical Peak Pricing Schemes. Energies 2017, 10, 2065. [CrossRef]

36. Mirjalili, S. Moth-flame optimization algorithm: A novel nature-inspired heuristic paradigm. Knowl.-Based Syst. 2015, 89, 228-249. [CrossRef]

37. Yang, X.S.; Deb, S. Cuckoo search via Lévy flights. In Proceedings of the World Congress on Nature \& Biologically Inspired Computing, Coimbatore, India, 9-11 December 2009; pp. 210-214.

38. Aslam, S.; Javaid, N.; Khan, F.A.; Alamri, A.; Almogren, A.; Abdul, W. Towards efficient energy management and power trading in a residential area via integrating grid-connected microgrid. Sustainability 2018, 10, 1245. [CrossRef]

39. Yang, X.S. Firefly algorithms for multimodal optimization, in Stochastic Algorithms: Foundations and Applications. Lect. Notes Comput. Sci. 2009, 5792, 169-178.

40. Cirocco, L.; Belusko, M.; Bruno, F. Optimization of Storage for Concentrated Solar Power Plants. Challenges 2014, 5, 473-503. [CrossRef]

(c) 2019 by the authors. Licensee MDPI, Basel, Switzerland. This article is an open access article distributed under the terms and conditions of the Creative Commons Attribution (CC BY) license (http:/ / creativecommons.org/licenses/by/4.0/). 